\title{
Crusted Umbilicated Papules in a Child
}

\author{
Hitaishi Mehta $^{1}$, Sheetanshu Kumar ${ }^{1}$, Divya Aggarwal ${ }^{2}$, Debajyoti Chatterjee ${ }^{2}$, \\ Keshavamurthy Vinay ${ }^{1}$
} 1 Department of Dermatology, Venereology and Leprology, Postgraduate Institute of Medical Education and Research, Chandigarh, India
2 Department of Histopathology, Postgraduate Institute of Medical Education and Research, Chandigarh, India

Key words: reactive perforating collagenosis, RPC, perforating disorders

Citation: Mehta H, Kumar S, Aggarwal D, Chatterjee D, Vinay K. Crusted umbilicated papules in a child. Dermatol Pract Concept. 2022;12(1):e2022002. DOI: https://doi.org/10.5826/dpc.1201a02

Accepted: April 14, 2021; Published: January 2022

Copyright: $\odot 2022$ Mehta et al. This is an open-access article distributed under the terms of the Creative Commons AttributionNonCommercial License (BY-NC-4.0), https://creativecommons.org/licenses/by-nc/4.0/, which permits unrestricted noncommercial use, distribution, and reproduction in any medium, provided the original authors and source are credited.

Funding: None.

Competing interests: None.

Authorship: All authors have contributed significantly to this publication.

Corresponding author: Keshavamurthy Vinay, MD, DNB, MNAMS, Department of Dermatology, Venereology and Leprology, Postgraduate Institute of Medical Education and Research, Chandigarh, India. E-mail: vinay.keshavmurthy@gmail.com.

\section{Introduction}

Reactive perforating collagenosis (RPC) is a rare benign perforating disorder characterized by transepidermal elimination of collagen fibers. Both familial and acquired forms of RPC exist, the latter in association with end-stage chronic kidney disease and diabetes mellitus. The very rare childhood form has slight male predilection and is commonly familial [1].

\section{Case Presentation}

Dermatology consultation was sought for a school-age child with multiple mildly itchy, red, raised lesions on his face and dorsum of hands, which were first noticed by the parents at 2 years of age. The parents also reported spontaneous healing of a few of the lesions with residual hypopigmented scars. Preceding history of insect bites or trauma was denied. $\mathrm{He}$ was born out of a non-consanguineous marriage, and no one in family had a history of similar lesions. Examination revealed multiple skin-colored to erythematous discrete papules on both cheeks (Figure 1A) and dorsum of hands (Figure 1B) with central adherent keratotic plugs along with few well-circumscribed areas of atrophic scars. Dermoscopy of an individual lesion is presented in Figure 2A. Routine laboratory parameters including renal function tests, fasting blood sugar, and urinalysis were within normal limits. Histopathological examination of a facial papular lesion revealed compact ortho- and parakeratotic epidermis with a cup-shaped lesion lined by acanthotic epidermis on both sides along with mild perivascular and periadnexal lymphomononuclear cell infiltrate mixed with collagen bundles in the dermis (Figure 2B). Altered collagen could be seen extruding trans epidermally through the cup-shaped depression. A diagnosis of RPC was reached. The child was treated with topical tretinoin $0.025 \%$ ointment with satisfactory improvement after 1 month.

\section{Conclusions}

Familial RPC commonly presents as erythematous papules with central adherent scale-crust over the extensor aspect of 


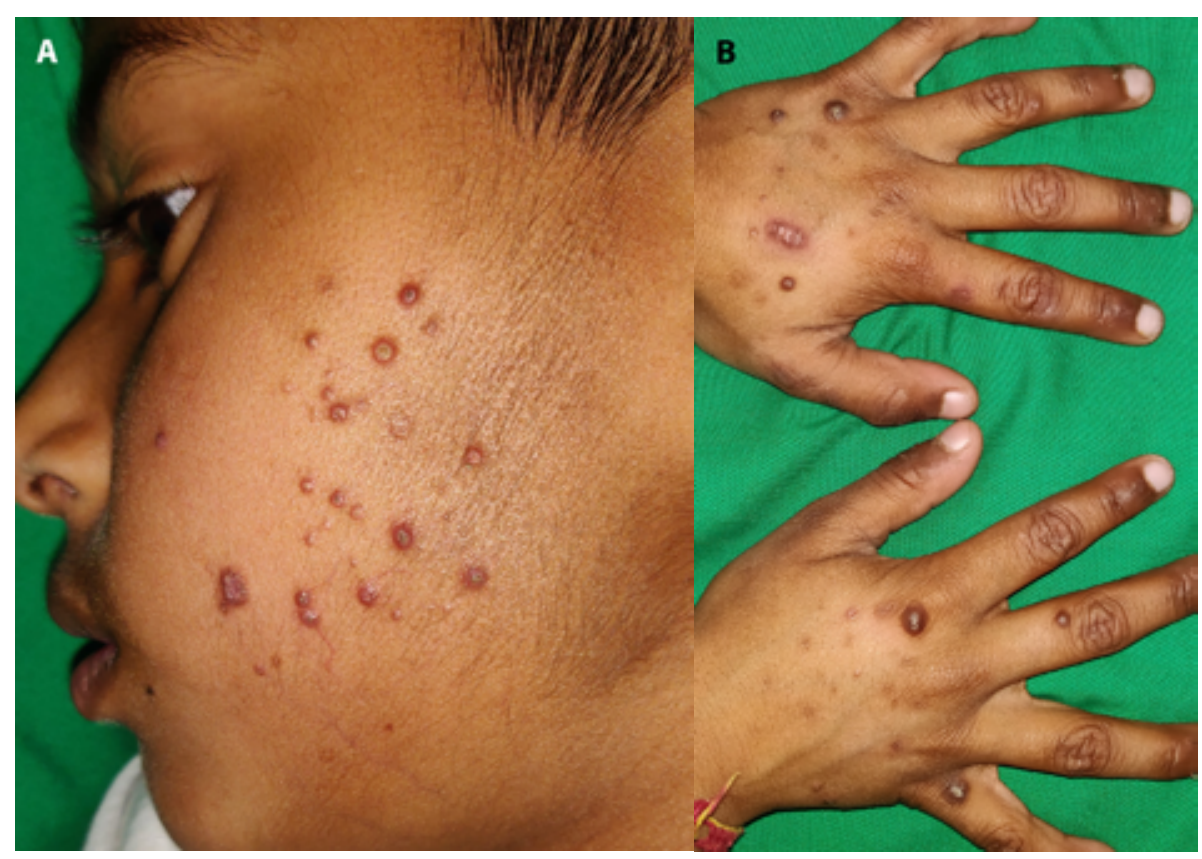

Figure 1. (A) Multiple skin-colored to erythematous discrete papules on left cheek with central adherent keratotic plugs along with few well circumscribed areas of atrophic scars on cheek. (B) Crusted umbilicated papules over dorsum of both hands.

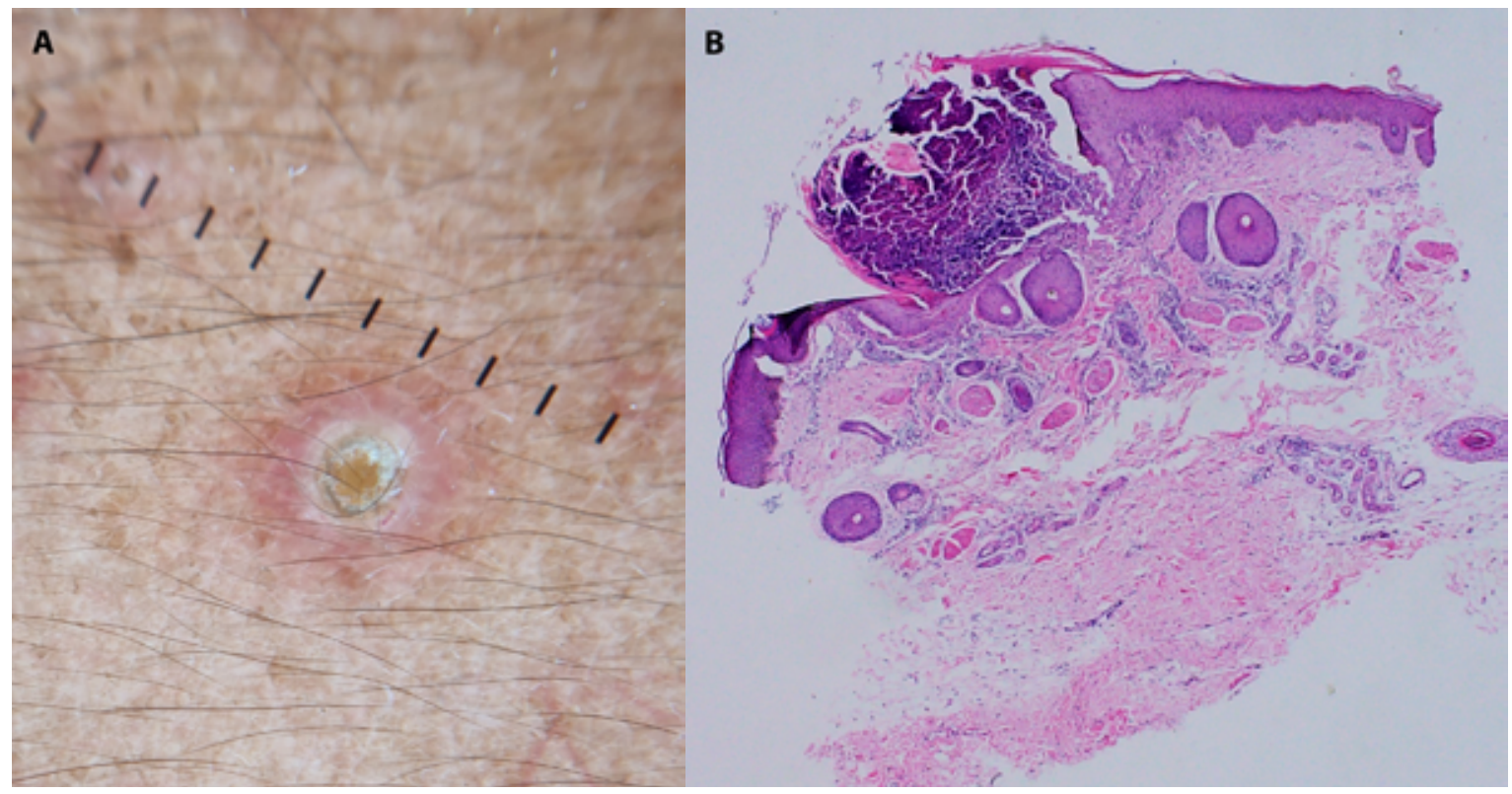

Figure 2. (A) Dermoscopy reveals a yellowish central homogenous area along with a white rim surrounded by erythematous halo at the periphery (DermLite II, Hybrid M, $\times 10$, polarized). (B) Histopathological examination (H\&E, $\times 40)$ of the punch biopsy taken from one of the papular lesions on the face showing orthokeratotic epidermis with a cup shaped lesion lined by acanthotic epidermis on both sides along with mild perivascular and periadnexal lymphomononuclear cell infiltrate mixed with collagen bundles in the dermis. The cup-shaped lesion was densely packed with degenerated basophilic collagen bundles.

extremities. Superficial trauma precedes most lesions. Individual lesions heal within 6-10 weeks, but recurrences are common. Although the familial and acquired forms appear similar in morphology and on histopathology, the acquired form tends to be more persistent and shows transepidermal elimination of both collagen and elastin.

The central homogeneous yellowish area observed on dermoscopy corresponds to the central scale or crust, and the white rim of varying thickness corresponds to the epidermal invagination, along with an erythematous halo which arises due to small blood vessels around the lesion [2]. Histopathology reveals a plug of keratotic debris with vertically oriented collagen fibers extending into the plug, along with parakeratosis, epithelial hyperplasia, and dyskeratotic keratinocytes [1].

The diagnosis is mostly clinical and requires histopathology for confirmation. Investigations other than punch 
biopsy are neither necessary nor contributory. Differential diagnosis includes papular urticaria, prurigo nodularis, perforating folliculitis, and elastosis perforans serpiginosa.

Along with avoidance of trauma, topical therapies reported to be successful include retinoids, corticosteroids, and salicylic acid. Other therapies are systemic antihistamines, retinoids, methotrexate, photochemotherapy, NB-UVB, and cryotherapy. Most of the patients have a relapsing-remitting course throughout their lifetimes.

Childhood RPC is a rare entity with an obscure etiopathogenesis and usually self-limiting course. Awareness among clinicians regarding this entity is imperative in order to avoid misdiagnosis, anxiety among parents, and over-aggressive management.
Informed consent: Informed consent for publication of clinical details and clinical images was obtained from the patient.

\section{References}

1. Millard PR, Young E, Harrison DE, Wojnarowska F. Reactive perforating collagenosis: light, ultrastructural and immunohistological studies. Histopathology. 1986;10(10):1047-1056. DOI:10.1111/j.1365-2559.1986.tb02541.x. PMID: 2430879.

2. Kittisak P, Tanaka M. Dermoscopic findings in a case of reactive perforating collagenosis. Dermatol Pract Concept. 2015;5(2): 75-77. DOI: 10.5826/dpc.0502a13. PMID: 26114057. PMCID: PMC4462904. 